\title{
Geant4 simulation of the response of phosphor screens for X-ray imaging
}

\author{
S.A. Pistrui-Maximean ${ }^{\mathrm{a}, *}$, N. Freud $^{\mathrm{a}}$, J.M. Létang ${ }^{\mathrm{a}}$, A. Koch $^{\mathrm{b}}$, \\ B. Munier ${ }^{\mathrm{b}}$, A.H. Walenta ${ }^{\mathrm{c}}$, G. Montarou ${ }^{\mathrm{d}}$ D. Babot ${ }^{\mathrm{a}}$, \\ ${ }^{a}$ Laboratory of Nondestructive Testing using Ionizing Radiation, \\ INSA-Lyon Scientific \& Technical University, Bât. Antoine de Saint Exupéry, \\ 69621 Villeurbanne Cedex, France \\ ${ }^{\mathrm{b}}$ Thales Electron Devices, 38430 Moirans, France \\ ${ }^{\mathrm{c}}$ Department of Detectors and Electronics, FB Physik, University of Siegen, \\ 57068 Siegen, Germany \\ ${ }^{\mathrm{d}}$ Corpuscular Physics Laboratory, Blaise Pascal University, \\ 63177 Aubière Cedex, France
}

\begin{abstract}
In order to predict and optimize the response of phosphor screens, it is important to understand the role played by the different physical processes inside the scintillator layer. Monte Carlo simulations were carried out to determine the Modulation Transfer Function (MTF) of phosphor screens for energies used in X-ray medical imaging and nondestructive testing applications. The visualization of the dose distribution inside the phosphor layer gives an insight into how the MTF is progressively degraded by X-ray and electron transport. The simulation model allows to study the influence of physical and technological parameters on the detector performances, as well as to design and optimize new detector configurations. Preliminary MTF measurements have been carried out and agreement with experimental data has been found in the case of a commercial screen (Kodak Lanex Fine), at an X-ray tube potential voltage of $100 \mathrm{kV}$. Further validation with other screens (transparent or granular) at different energies is under way.
\end{abstract}

Key words: Monte Carlo simulation, Geant4, phosphor screen, X-ray imaging, Modulation Transfer Function

PACS: 07.05.Tp

* Corresponding author.

Email address: simona.pistrui@insa-lyon.fr (S.A. Pistrui-Maximean). 


\section{Introduction}

Because of their X-ray detection and image resolution properties, phosphor screens have been employed in a large number of applications for X-ray imaging. Most commercial systems use a silicon Charged Coupled Device (CCD) coated with granular $\mathrm{Gd}_{2} \mathrm{O}_{2} \mathrm{~S}(\mathrm{~Tb})$ or vapor-grown $\mathrm{CsI}(\mathrm{Tl})$ scintillators. A new scintillator material, europium doped lutetium oxide - $\mathrm{Lu}_{2} \mathrm{O}_{3}(\mathrm{Eu})$, in granular $[1,2]$ or in transparent optical ceramic (TOC) form [3], presents interesting properties: a high density of $9.4 \mathrm{~g} / \mathrm{cm}^{3}$ in TOC form, high light output comparable to $\mathrm{CsI}(\mathrm{Tl})$ and a close match to the maximum response of the CCD. Other approaches concentrate on developing innovative detector architectures, like $\mathrm{CsI}(\mathrm{Tl})$-filled pores created in a silicon matrix, coated with an oxide $\left(\mathrm{SiO}_{2}\right)$ or a metal $(\mathrm{Ru}, \mathrm{Pt})$ layer and combined with a CCD detector [4-6]. In order to predict and optimize the response of phosphor screens, analytical models have been developed, notably by Swank [7]. Other approaches use Monte Carlo techniques [8], which provide an accurate simulation of radiation transport in fluorescent screens due to the random nature of the physical phenomena. In this paper, we propose a simulation model based on the Monte Carlo code Geant4 [9] for the prediction and optimization of the spatial resolution properties of fluorescent screens.

\section{Simulation principle}

The phosphor screen response is simulated with the Geant 4 code in the case of a pencil beam of monochromatic X-ray photons impinging orthogonally onto the scintillator (Fig. 1). An ideal detector situated behind the screen records all the impacts of the optical photons coming out of the scintillator. The light distribution under pencil beam excitation represents the Point Spread Function of the scintillator screen, $\operatorname{PSF}(x, y)$. By integrating $\operatorname{PSF}(x, y)$ over the $y$-coordinate, one obtains the Line Spread Function, $\operatorname{LSF}(x)$. The screen modulation transfer function, $\operatorname{MTF}(\nu)$, is the one dimensional Fast Fourier Transform (FFT) of $\operatorname{LSF}(x)$.

$$
\begin{gathered}
\operatorname{LSF}(x)=\int \operatorname{PSF}(x, y) \mathrm{d} y \\
\operatorname{LSF}(x) \stackrel{\mathrm{FFT}}{\longrightarrow} \operatorname{MTF}(\nu)
\end{gathered}
$$

The influence of statistics (number of incident photons) and of sampling parameters (pixel size of the virtual ideal detector and detector dimensions) on the simulated MTF has been studied, to ensure the convergence of the results. 


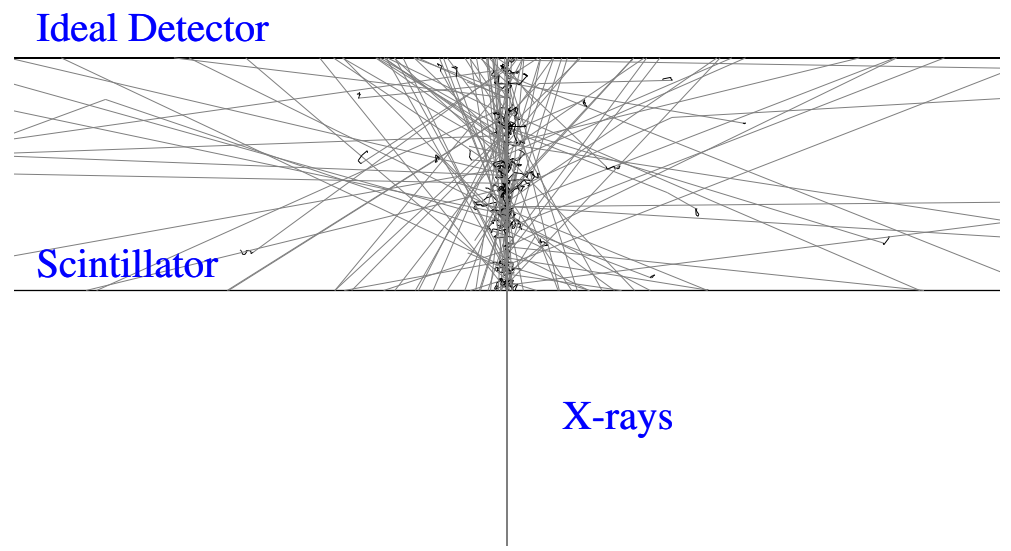

Fig. 1. Geant4 simulation of $10^{3}$ incident X-ray photons with an energy of $65 \mathrm{keV}$ orthogonally impinging onto a $84 \mu \mathrm{m}$ thick $\mathrm{Gd}_{2} \mathrm{O}_{2} \mathrm{~S}$ powder scintillator. X-ray tracks are represented in gray and electron tracks in black.

\section{$3 \quad$ Effect of X-ray and electron processes}

The role played by X-ray and electron interactions was analyzed by activating progressively the physics processes and by visualizing the distribution of deposited energy in the scintillator (Fig. 2). The simulated processes are: photoelectric effect, Compton and Rayleigh scattering, for the X-ray photons, ionization, multiple scattering and bremsstrahlung, for the electrons, and the de-excitation of the atom by fluorescence and Auger effect. X-ray and electron processes both degrade the spatial resolution. In a first approach, scintillation photons are considered to propagate in the phosphor without absorption, scattering or reflection. The Geant4 simulation results compare well with Swank's analytical MTF [7], derived in the case of a transparent phosphor when visible photons are generated only by exponential X-ray absorption in the phosphor, with no reflective backing layer (Fig. 3). It is noteworthy that Monte Carlo simulations make it possible to study the role played by physical interactions not taken into account by Swank's model.

\section{Role played by optical processes and experimental validation of the simulation results}

An optical model has been used in which the phosphor layer is considered as a homogeneous medium with macroscopic absorption and scattering coefficients (scattering is considered to be isotropic). The values of the absorption and scattering coefficients of optical photons were taken to be equal to $2.42 \mathrm{~mm}^{-1}$ and $23.79 \mathrm{~mm}^{-1}$, according to previous studies [8]. The optical processes at the interface between two media (reflection, refraction) are not considered yet. 


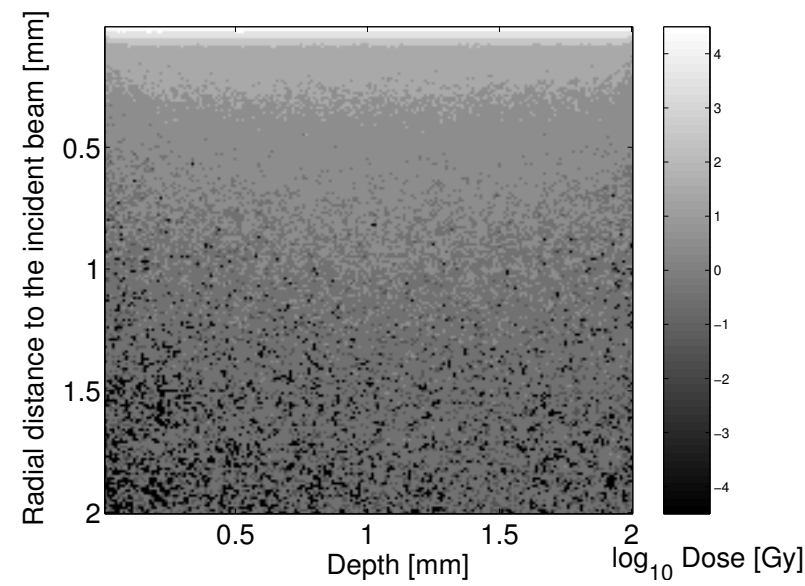

Fig. 2. Distribution of the dose deposited by a $300 \mathrm{keV}$ pencil beam of $9 \times 10^{5}$ photons orthogonally impinging onto a $2 \mathrm{~mm}$ thick $\mathrm{Gd}_{2} \mathrm{O}_{2} \mathrm{~S}$ crystal.

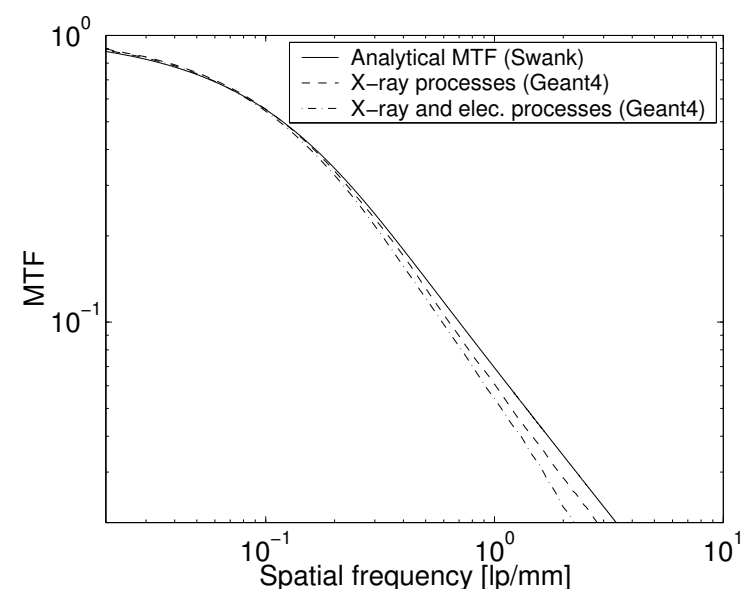

Fig. 3. Comparison between Geant4 simulated MTFs and Swank's analytical model in the case of a $300 \mathrm{keV}$ pencil beam of $1.5 \times 10^{4}$ photons normally impinging onto a $2 \mathrm{~mm}$ thick $\mathrm{Gd}_{2} \mathrm{O}_{2} \mathrm{~S}$ crystal. The scintillator spatial resolution is progressively degraded by X-ray and electron processes.

The complete scintillator structure (backing layer, substrate, phosphor layer and overcoat) has been simulated.

In order to validate the simulation model, MTF measurements have been carried out using a $50 \mu \mathrm{m}$ wide uranium slit superimposed on a commercial scintillator (Kodak Lanex Fine, $84 \mu \mathrm{m}$ thick granular $\mathrm{Gd}_{2} \mathrm{O}_{2} \mathrm{~S}(\mathrm{~Tb})$ layer, 8 $\mu \mathrm{m}$ overcoat). The slit-scintillator combination was irradiated by a $\mathrm{W}$-target $\mathrm{X}$-ray tube at $100 \mathrm{kV}$ and filtered by a $22 \mathrm{~mm}$ thick $\mathrm{Al}$ plate. The images were acquired using a low noise CCD camera (1024 pixels $\times 1024$ pixels of $24 \mu \mathrm{m}$ in size, $14 \mathrm{bits})$ cooled at $-40^{\circ} \mathrm{C}$. The images were magnified with a $55 \mathrm{~mm}$ Nikon optics so that the pixel size in the object plane was $7.4 \mathrm{\mu m}$. In the slit method $[11,12]$, the experimental LSF represents the convolution of the PSF with the slit. The MTF is calculated by taking the Fourier transform of the LSF. The simulated MTF was compared with the experimental results 
in the case of the Kodak Lanex Fine screen (Fig. 4). The degradation of the measured MTF due to the finite slit width and camera response was assumed to be negligible. It appears that the optical processes improve the scintillator spatial resolution in comparison with a transparent phosphor.

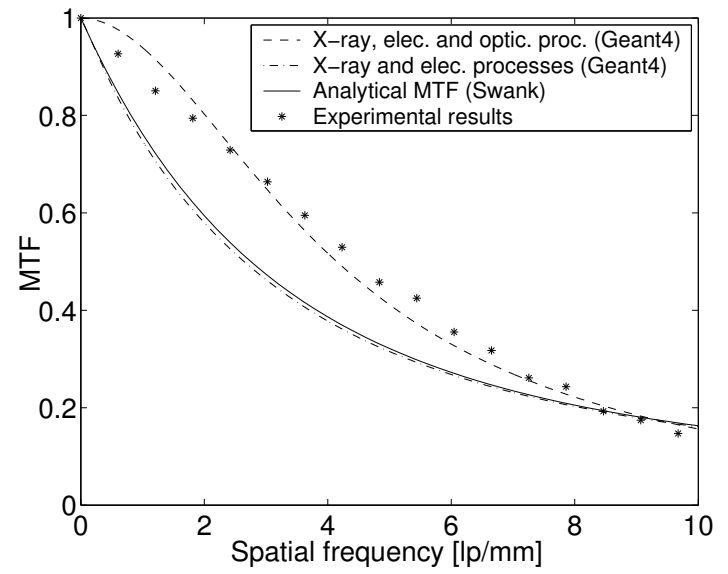

Fig. 4. Comparison between simulated and experimental MTFs for a $100 \mathrm{kV}$ X-ray beam filtered by a $22 \mathrm{~mm}$ thick $\mathrm{Al}$ plate.

As confirmed by the simulation results, the protective overcoat layer situated between the granular phosphor layer and the detector of visible photons degrades the spatial resolution due to a geometrical effect (Fig. 5).

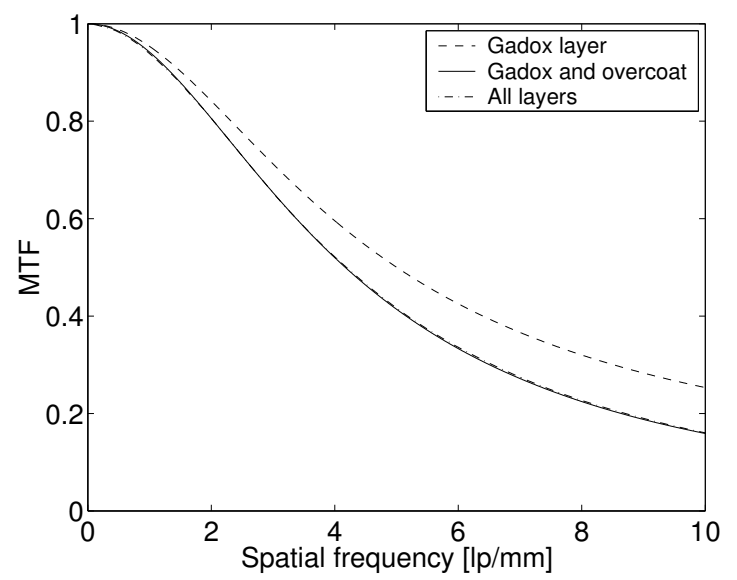

Fig. 5. Simulated MTFs show the degradation caused by the protective overcoat layer situated between the granular phosphor layer and the detector of visible photons, in the case of the Kodak Lanex Fine scintillator. The degradation is due to a geometrical effect.

\section{Conclusions and future work}

A simulation model has been developed in order to study the response of phosphor screens for X-ray imaging. The simulation results are in agreement 
with the preliminary measurements in the case of Kodak Lanex Fine screen. Further validation of our model with other screens (transparent or granular) at different energies is under way. The proposed model still has to be refined as regards the optical photon transport, notably to account for surface interactions. Further measurements will be carried out to assess the role of optical phenomena in greater details. The simulation model can be used as a tool to optimize existing detector configurations (e.g. by introducing reinforcing metallic screens or by optimizing light absorption in the scintillator) and may help in designing new detector architectures.

\section{References}

[1] C. Dujardin, A. Garcia-Murillo, C. Pedrini, C. Madej, C. Goutaudier, A. Koch, A.G. Petrosyan, K.L. Ovanesyan, G.O. Shirinyan, and M.J. Weber. Synthesis and scintillation properties of some dense X-ray phosphors. In: Proceedings of the 5th International Conference on Inorganic Scintillators and Their Applications (SCINT99), 16-20 August 1999, Moscow, Russia, 1:527-531, 1999.

[2] V.V. Nagarkar, S.R. Miller, S.V. Tipnis, A. Lempicki, C. Brecher, and H. Lingertat. A new large area scintillator screen for X-ray imaging. Nuclear Instruments and Methods in Physics Research B, 213:250-254, 2004.

[3] A. Lempicki, C. Brecher, P. Szupryczynski, H. Lingertat, V.V. Nagarkar, S.V. Tipnis, and S.R. Miller. A new lutetia-based ceramic scintillator for X-ray imaging. Nuclear Instruments and Methods in Physics Research A, 488:579590, 2002.

[4] X. Badel, A. Galeckas, J. Linnros, P. Kleimann, C. Frojdh, and C.S. Petersson. Improvement of an X-ray imaging detector based on a scintillating guides screen. Nuclear Instruments and Methods in Physics Research A, 487:129-135, 2002 .

[5] X. Badel, J. Linnros, M.S. Janson, and J. Osterman. Formation of pn junctions in deep silicon pores for X-ray imaging detector applications. Nuclear Instruments and Methods in Physics Research A, 509:96-101, 2003.

[6] Xavier Badel. Development of macropore arrays in silicon and related technologies for X-ray imaging applications. PhD Thesis in Materials and Semiconductor Physics (1st part), Stockholm: KTH, Royal Institute of Technology, integrated part of 3D Radiation Imaging Detectors (3D-RID) European Project, 2003, 44 p.

[7] Robert K. Swank. Calculation of modulation transfer functions of X-ray fluorescent screens. Applied Optics, 12(8):1865-1870, 1973.

[8] P.F. Liaparinos, I.S. Kandarakis, D.A. Cavouras, D.N. Nikolopoulos, and G.S. Panayiotakis. Simulating the absorption efficiency and resolution properties 
of fluorescent screens by Monte Carlo methods. In: IEEE Nuclear Science Symposium Conference Record, 16-22 Oct. 2004, Rome, Italy, 2:874-878, 2004.

[9] S. Agostinelli et al. Geant4 - a simulation toolkit. Nuclear Instruments and Methods in Physics Research A, 506(3):250-303, 2003.

[10] R.M. Nishikawa and M.J. Yaffe. Model of the spatial-frequency-dependent detective quantum efficiency of phosphor screens. Medical Physics, 17:894-904, 1990.

[11] H. Fujita, D.Y. Tsai, T. Itoh, K. Doi, J. Morishita, K. Ueda, and A. Ohtsukn. A simple method for determining the modulation transfer function in digital radiography. IEEE Transactions on Medical Imaging, 11(1):34-39, 1992.

[12] John M. Boone et al. Handbook of Medical Imaging, volume 1. Physics and Psychophysics. SPIE Press, 2000. 